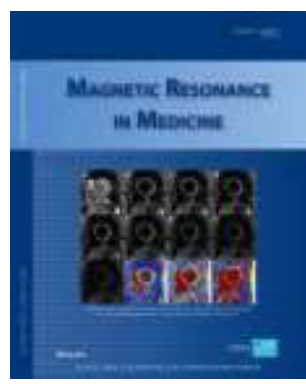

\title{
Simultaneous proteoglycans and hypoxia mapping of chondrosarcoma environment by frequency selective CEST MRI
}

\begin{tabular}{|r|l|}
\hline Journal: & Magnetic Resonance in Medicine \\
\hline Manuscript ID & MRM-20-21766.R1 \\
\hline Wiley - Manuscript type: & Full Paper \\
\hline Research Type: & Physiological Research, Translational Research < Physiological Research \\
\hline Research Focus: & Cancer, Pathology < Function < Cartilage < Musculoskeletal \\
\hline
\end{tabular}

\section{SCHOLARONE" \\ Manuscripts}


Roxane Autissier ${ }^{1,2,3}$; Leslie Mazuel ${ }^{1,2,3}$; Elise Maubert ${ }^{2}$; Jean-Marie Bonny ${ }^{1,3}$; Philippe Auzeloux ${ }^{2}$;

4

Sébastien Schmitt²; Amidou Traoré1,3; Caroline Peyrode ${ }^{2}$; Elisabeth Miot-Noirault ${ }^{2} \&$ Guilhem $^{2}$

5

Pagès ${ }^{1,3}$

6

$7 \quad{ }^{1}$ INRAE, UR QuaPA, F-63122 Saint-Genès-Champanelle, France

8 2Université Clermont Auvergne, Inserm, IMoST, F-63000 Clermont-Ferrand, France

$9 \quad{ }^{3}$ AgroResonance, INRAE, 2018. Nuclear Magnetic Resonance Facility for agronomy, food and health, 10 https://doi.org/10.15454/1.5572398324758228E12 11

Correspondence: Roxane Autissier, PhD student

13 UMR 1240 IMoST Inserm/Université Clermont Auvergne

14 58, rue Montalembert, 63005 Clermont Ferrand cedex, France

15 e-mail : Roxane.AUTISSIER@uca.fr

16

17

Grant: Ligue Contre le Cancer Auvergne - Rhône - Alpes. 18

Word count: 4201

20 21 


\section{ABSTRACT}

24

25

26

27

28

29

30 31

Purpose: To evaluate the relevance of CEST frequency selectivity in simultaneous in vivo imaging of both of chondrosarcoma's (CHS) phenotypic features, i.e. its high proteoglycan (PG) concentration and its hypoxic core.

Methods: Swarm rat CHSs were implanted subcutaneously in NMRI Nude mice. When tumors were measurable (between 12 and 16 days post-operative), mice were submitted to GAG, guanidyl and amide proton transfer (APT) CEST imaging. PGs and hypoxia were assessed in parallel by nuclear imaging exploiting ${ }^{99 \mathrm{~m} T c-N T P} 15-5$ and ${ }^{18} \mathrm{~F}-\mathrm{FMISO}$, respectively. Data were completed by ex vivo analysis of PGs (histology and biochemical assay) and hypoxia (immunofluorescence).

Results: Quantitative analysis of GAG CEST evidenced a significantly higher signal for tumor tissues than for muscles. These results were in agreement with nuclear imaging and ex vivo data. For imaging tumoral $\mathrm{pH}$ in vivo, the CEST ratio of APT/Guanidyl was studied. It highlighted an important heterogeneity inside the tumor. The hypoxic status was confirmed by ${ }^{18} \mathrm{~F}-\mathrm{FMISO}$ TEP imaging and ex vivo immunofluorescence.

Conclusion: CEST MRI simultaneously imaged both CHS properties during a single experimental run and without the injection of any contrast agent. Both MR and nuclear imaging as well as ex vivo data were in agreement and showed that this CHS animal model was rich in PGs. However, even if tumors were lightly hypoxic at the stage studied; acidic areas were highlighted and mapped inside the tumor.

Keywords: CEST; Chondrosarcoma; Hypoxia; MRI; Nuclear imaging; Proteoglycans. 


\section{INTRODUCTION}

Chondrosarcoma (CHS) is a malignant cartilaginous tumor representing the most common primary bone cancer in adults. ${ }^{(1,2)}$ CHS is characterized by a dense heterogeneous extracellular matrix (ECM), a low percentage of dividing cells and poor vascularity. ${ }^{(3)}$ The combination of these three factors leads to chemo and radiation therapy resistance. ${ }^{(4-8)}$

Dense and fibrotic ECM with high levels of collagen and proteoglycans (PGs) is recognized to impair interstitial transport of drug from blood to cancer cells. ${ }^{(9)}$ In addition, the acidic microenvironment associated with hypoxia may electrostatically charge the drugs, limiting their ability to cross biological membranes.(10) In vivo assessment of the phenotypic topological heterogeneity of the tumor microenvironment is key to optimizing personalized therapies. Although, biopsy is subject to sampling and pathologist/observer variability, microenvironmental characterization is mainly achieved from biopsy samples to determine grade and staging of the disease. ${ }^{(11)}$ Tumor evaluation requires access to morphological and protein information in a context that takes into account intratumoral heterogeneity as a whole. In such a situation, developing imaging methods to access the main recognized CHS features, i.e. chondrogenic matrix and hypoxia, would be of great interest for diagnosis and clinical follow-up. Indeed, the current clinical imaging methods recommended, i.e. CT and MRI, ${ }^{(12)}$ are used to define tumor morphology and distinguish postoperative residues from postoperative or post-chemotherapeutic residual lesions. However, such approaches cannot be used in the same manner as functional imaging to assess tumor environment. ${ }^{(13)}$

Functional imaging can be done with specific radiotracers to access metabolic data useful to diagnose and assess the therapeutic response of CHS. Due to their rarity, there is a lack of large trials on functional imaging of chondroid tumors. Nowadays, nuclear medicine used to image CHS in a clinical context targets either the bone remodeling or the glucose tumoral metabolism with radiotracers such as ${ }^{99 \mathrm{~m}} \mathrm{Tc}-\mathrm{MDP}$ or ${ }^{18} \mathrm{~F}-\mathrm{FDG}$, respectively. ${ }^{(14)}$ To more accurately image the CHS microenvironment, radiotracers should target either ECM PGs or the CHS hypoxia feature. ${ }^{99 \mathrm{~m} T c-N T P} 15-5$ emerged from our lab as a candidate SPECT tracer for the functional imaging of cartilage by targeting PG negative charges in ECM. It demonstrated its relevance to specifically diagnose on CHS animal model and is expected to enter clinical trials shortly. ${ }^{(15-17)}$ One of the radiotracers extensively used in clinic for hypoxia is ${ }^{18} \mathrm{~F}$-FMISO. ${ }^{(18)}$ As with many nitroimidazole derivatives, under reduced oxygen conditions, this tracer is reduced into a $\mathrm{R}-\mathrm{NO}_{2}$ radical that binds covalently to intracellular molecules leading ultimately to the entrapment of the tracer in hypoxic cells. To image both tumor features, two nuclear exams are required with two injections of radioactive contrast agent. Therefore, it would be interesting to develop a method to simultaneously image both of the metabolic properties of CHS.

The recent rise of CEST MRI has opened up new perspectives in the imaging of biological molecules within the tumoral microenvironment. ${ }^{(19)}$ This method is based on the exchange between saturated labile protons of the solute with the bulk water, revealing the endogenous contrast of some chemical functions. CEST MRI indirectly maps these low concentration metabolites by monitoring the variation of an 
elevated water signal. Typically, a CEST experiment is a 2-step process. First, the magnetic field inhomogeneity has to be mapped; this is the function of the so-called water saturation shift referencing (WASSR) acquisition. Second, MR images must be recorded at several saturation frequency offsets to record a z-spectrum (normalized water signal as a function of the saturation offset for each voxel). This z-spectrum, with a zero-frequency referenced to the water's frequency will display an extra water attenuation at the metabolite exchangeable proton frequency, i.e. the CEST effect. To quantify this CEST effect, the first proposed method was based on the analysis of the water signal intensity at the offset of interest and its counterpart negative value. This magnetization transfer ratio asymmetry (MTRasym) analysis hypothesizes the absence of the magnetization transfer effect for negative offsets. As this hypotheses is not systematically verified, other z-spectrum analyses were developed, i.e. a threeoffset measurement approach or multiple-pool Lorentzian fitting method. ${ }^{(20,21)}$ Hydroxyl, guanidyl and amide moieties were independently imaged by CEST MRI. ${ }^{(22,23)}$ Readers interested in a deeper explanation of CEST MRI principles are referred to the following references. ${ }^{(24-26)}$

CEST MRI has demonstrated its relevance for the quantitative evaluation and mapping of PGs in cartilaginous tissues by assessing the chemical exchange of saturable protons with the hydroxyl groups (i.e., around $0.5-1.5 \mathrm{ppm}$ ) of GAG molecules ${ }^{(27,28)}$ in both physiological and pathological situations. ${ }^{(29-}$ 31) In addition, CEST MRI has been demonstrated to be sensitive to $\mathrm{pH}$ variations, indirectly revealing information on the metabolic switch in response to hypoxia. ${ }^{(32)}$ Several studies have demonstrated that $\mathrm{pH}$ variations impact the chemical exchange rate of both guanidyl and amide moieties. ${ }^{(33,34)}$ The change in the chemical exchange rate with the $\mathrm{pH}$ leads to a variation of the CEST intensity at frequencies of around $1.8-2.4$ and $3.0-4.0 \mathrm{ppm}$ for guanidyl and amide proton transfer (APT) CEST, respectively. Therefore, the combination of both guanidyl and APT CEST has been proposed as a highly sensitive method to explore extracellular $\mathrm{pH}$ acidification in tumors. ${ }^{(35,36)}$

Considering that $\mathrm{PGs}$ and $\mathrm{pH}$ each have their own resonance frequencies, and are therefore sensitive to different exchangeable moieties, we decided these two features would merit further study using a single CEST MRI acquisition on CHS animal model. The aim of this study was to evaluate the relevance of frequency-selected CEST MRI to simultaneously map PG concentration and $\mathrm{pH}$ of CHS tissue in vivo. To validate this approach, CEST MRI was compared to the standard reference nuclear imaging as well as ex vivo analysis.

\section{METHODS}

\subsection{GAG and $\mathrm{pH}$ phantom experiments}

Phosphate-buffered saline (PBS) 1X (Gibco Laboratories, Gaithersburg, MD, USA) containing 1\% of bovine serum albumin (BSA) solution (Sigma Aldrich, St. Louis, MO, USA) was used to prepare the chondroitin 4-sulfate phantom. Five samples of the chondroitin 4-sulfate (Sigma Aldrich, St. Louis, MO, USA) were prepared at. $1 ; 5 ; 10 ; 20 ; 40 \mathrm{mg} / \mathrm{mL}$ at $\mathrm{pH} 7.4$. For protamine phantom, $15 \mathrm{mg} / \mathrm{mL}$ of protamine (Sigma Aldrich, St. Louis, MO, USA) and $0.15 \mathrm{mM}$ of $\mathrm{MnCl}_{2}$ were dissolved in PBS. Four 
118

119

120

121

122

123

124

125

126

127

128

129

130

131

132

133

134

135

136

137

138

139

140

141

142

143

144

145

146

147

148

149

150

151

152

153

samples of protamine were prepared with $\mathrm{pH}$ ranging from 6.5 to 7.7 in 0.4 unit increments. Both phantoms (chondroitin 4-sulfate and protamine) were prepared with $15 \%$ gelatin in water to fix the syringes containing the different solutions. Phantoms were submitted to the same acquisition and analysis protocols as described below except for $\mathrm{B}_{1}$, which was set to $3 \mu \mathrm{T}$.

\subsection{Xenograft model of SWARM rat CHS}

Experiments were conducted in accordance with the European directive 2010/63/EU after approval by the animal ethical committee C2E2A, authorization number: \#15991.

SWARM rat CHS (SRC) xenografts were performed in 5 week old female NMRI Nude immunodeficient mice (Janvier Labs, Le Genest-Saint-Isle, FR) from frozen fragments after reactivation in 4 week old male Sprague Dawley rats (Charles River Laboratories France, Saint Germain Nuelles, FR). For reactivation, SRC fragments were defrosted in Dulbecco's Modified Eagle Medium (DMEM, Gibco Laboratories, Gaithersburg, MD, USA) then implanted subcutaneously on the right flank of 3 anesthetized rats (isoflurane; $2.5 \%, 1 \mathrm{~L} / \mathrm{min}$, air $/ \mathrm{O}_{2}, 70 / 30, \mathrm{v} / \mathrm{v}$ ). After 22 days post-surgery, tumors were resected and immediately placed in cold DMEM. Then, they were manually calibrated to $1 \mathrm{~mm}^{3}$ and transplanted subcutaneously between the shoulder blades on anesthetized (isoflurane; $1.5 \%, 1$ $\mathrm{L} / \mathrm{min}$, air/ $\left.\mathrm{O}_{2}, 70 / 30, \mathrm{v} / \mathrm{v}\right)$ NMRI Nude immunodeficient mice $(\mathrm{n}=10)$ and cutaneous wounds were sutured.

\subsection{In vivo animal imaging}

All imaging experiments were performed at "In Vivo Imaging in Auvergne" (IVIA) facility (https://doi.org/10.18145/ivia). Imaging protocols were performed on the same mice between 12 and 16 days after induction, corresponding to an average tumoral volume of $215.04 \pm 86.28 \mathrm{~mm}^{3}$ (see supporting Figure S1). During these in vivo acquisitions, all animals were anesthetized by isoflurane $\left(1.5 \%, 1 \mathrm{~L} / \mathrm{min}\right.$, air/ $\left.\mathrm{O}_{2}, 70 / 30, \mathrm{v} / \mathrm{v}\right)$. All animals were secured on a dedicated bed with both breathing rate and temperature being monitored during acquisitions. Supporting Table S1 describes in details the animal distribution for each imaging modality.

\subsubsection{MR imaging}

MR images of mice $(n=10)$ were acquired on an 11.7 Tesla Bruker BioSpec system (Bruker BioSpin, Ettlingen, Germany) piloted by Paravision 6.0.1 software. A $72 \mathrm{~mm}$ circular polarizer volume coil was used for RF transmission and a surface array coil $(2 \times 2)$ positioned on both the tumor and the adjacent muscle received the NMR signal (Bruker BioSpin, Ettlingen, Germany).

$D W$ imaging: Tumor imaging was performed with a DWI sequence $\left(\mathrm{b}=500 \mathrm{~s} . \mathrm{mm}^{-2}\right.$, direction $[0,0,1])$ with a TE of $27 \mathrm{~ms}$ and a TR of $2000 \mathrm{~ms}$. The FOV was $40 \mathrm{~mm}$ with an axial slice thickness of $1 \mathrm{~mm}$ leading to a nominal resolution of $0.26 \times 0.26 \times 1 \mathrm{~mm}^{3}$. The number of slices was adjusted to 
154

155

156

157

158

159

160

161

162

163

164

165

166

167

168

169

170

171

172

173

174

175

176

177

178

179

180

181

182

183

184

185

186

187

188

189

190

image the whole tumor (generally, 12 slices were needed). Two averages were performed for a total acquisition time of $7 \mathrm{~min} 32 \mathrm{~s}$.

CEST Imaging: CEST acquisitions were based on a RARE sequence with an effective TE and TR of 4.756 and $4500 \mathrm{~ms}$, respectively and a RARE acceleration factor of 16 . The slice thickness was $2 \mathrm{~mm}$ and the FOV $40 \mathrm{~mm}$, i.e. the in-plane voxel size was $0.625 \times 0.625 \mathrm{~mm}^{2}$. Each image was recorded from a single transient. A first $\mathrm{z}$-spectrum was recorded to correct for $\mathrm{B}_{0}$ inhomogeneities by using the WASSR approach ${ }^{(37)}$ (saturation pulses $\mathrm{B}_{1} 0.1 \mu \mathrm{T}, 54.8 \mathrm{~ms}$ gaussian pulses repeated 18 times with an interpulse delay of a $0.010 \mathrm{~ms}$, and a bandwidth of $20 \mathrm{~Hz}$ leading to a train of $1 \mathrm{~s}$; saturation offsets from $\pm 1000 \mathrm{~Hz}$ with $\Delta \omega 20 \mathrm{~Hz}$ ) followed by the CEST z-spectrum (30 min acquisition duration with saturation pulses: $\mathrm{B}_{1} 1.5 \mu \mathrm{T}, 54.8 \mathrm{~ms}$ gaussian pulses repeated 72 times with a bandwidth of $50 \mathrm{~Hz}$ leading to a train of $4 \mathrm{~s}$ and an equivalent continuous wave CEST saturation of $0.6 \mu \mathrm{T}$; saturation offsets from $\pm 2500 \mathrm{~Hz}$ with $\Delta \omega 50 \mathrm{~Hz}$ ). CEST MRI data were analyzed with an in-house program written in MATLAB ${ }^{\circledR}$ R2017a (MathWorks, Natick, MA USA). In brief, a ROI was manually drawn on the highresolution DW image to delineate the tumor and muscle of the scapular region. Then, $\mathrm{B}_{0}$ shift was corrected pixel-by-pixel with the WASSR approach. Finally, the CEST methodology applied to analyze the data depended on the saturation offset. For GAG CEST, the analysis was performed on the whole image and the z-spectrum was fitted with two Lorentzian shapes: the first one to estimate the signal attenuation due to the water direct saturation (centered at $0 \mathrm{ppm}$ ) and the second one for the hydroxyl moieties. The GAG CEST signal intensity corresponded to the area of this latter Lorentzian shape. Regarding guanidyl and APT CEST, we applied the three-offset measurement approach proposed by Jin et al. ${ }^{(20)}$ to avoid nuclear overhauser enhancement (NOE) interferences. In practice, two limits were chosen (1.60 to 2.40 and 3.20 to $4.00 \mathrm{ppm}$ for the guanidyl and amide moieties, respectively) and a baseline was drawn between these frequencies. The CEST effect for APT* and guanidyl* were quantified as the difference between the baseline and the water signal at 1.96 and $3.68 \mathrm{ppm}$, respectively. The ratio of $\mathrm{APT}^{*} /$ guanidyl* was then calculated. The relative variation of this ratio was expressed from the mean ratio value on the ROI.

\subsubsection{Nuclear Imaging}

PGs imaging by SPECT/CT using ${ }^{99 m}$ Tc-NTP $15-5$ radiotracer: SPECT-CT images were acquired using a camera (NanoScan ${ }^{\circledR}$,Mediso Ltd, Budapest, Hungary) equipped with four multi pinhole detectors APT62. Acquisitions were performed on Nucline software (v3.00.018, Mediso Ltd, Budapest, Hungary). $20 \mathrm{MBq}$ of ${ }^{99 \mathrm{~m} T \mathrm{c}-\mathrm{NTP}} 15-5$, radiolabeled as described ${ }^{(38)}$, were injected intravenously in the tail vein. After 30 min, mice $(n=10)$ were anesthetized and imaged. Anatomic CT acquisition consisted of a helical scan with 480 projections, $300 \mathrm{~ms}$ per projection, $50 \mathrm{kV}, 590 \mu \mathrm{A}$. Then, SPECT images were performed with 128 projections, 20 s per projection and an energy window set at $140 \mathrm{keV} \pm 20 \%$. After image reconstructions using the 3D ordered subset expectation maximization (3D OSEM)

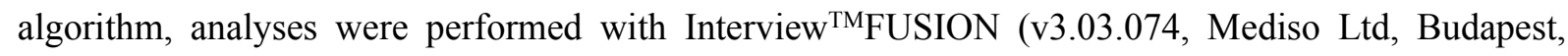


191 Hungary) software using an interpolated ellipse ROI for both tumor and scapular muscle manually drawn slice by slice. Activity concentrations were obtained in $\mathrm{kBq} / \mathrm{mL}$.

Hypoxia PET imaging using ${ }^{18} \mathrm{~F}$-FMISO: PET images were acquired using a small animal device (eXplore Vista ${ }^{\circledR}$, GE Healthcare, Chicago, IL, USA). $30 \mathrm{MBq}$ of ${ }^{18} \mathrm{~F}$-FMISO were injected intravenously in the tail vein. Four hours later, the mice $(n=10)$ were anesthetized and PET imaging was performed with a 250 to $700 \mathrm{keV}$ energy window and $6 \mathrm{~ns}$ coincidence time window (30 min duration, 2 bed positions) with MMWKS ${ }^{\circledR}$ Image software (GE Healthcare, Chicago, IL, USA). After 2D OSEM reconstructions, analyses were performed with VivoQuant ${ }^{\mathrm{TM}}$ (v4.0 patch1, inviCRO, Boston, MA, USA) software using a 3D ROI for tumor and scapular muscle manually drawn. ${ }^{18} \mathrm{~F}$-FMISO uptake was quantified in $\mathrm{kBq} / \mathrm{mL}$.

\subsection{Ex vivo analysis}

Histological and immunofluorescence analysis in tumoral tissues were performed at the histopathology facility ANIPATH (CNRS UMR 6293 / Inserm U1103 GReD, Clermont-Ferrand, FR).

After a multimodal imaging session, 20 days post-induction, mice $(n=3)$ were sacrificed by cervical dislocation under anesthesia and tumors were resected and cut into two samples. The first sample was fixed into $10 \%$ neutral buffered formalin for $24 \mathrm{~h}$ and embedded in paraffin for histological analysis (alcian blue or pimonidazole staining). The second sample was frozen in liquid nitrogen for GAG assays. For pimonidazole analysis, animals received an intraperitoneal injection of pimonidazole hydrochloride (60 mg/kg, Hypoxyprobe ${ }^{\mathrm{TM}}$, Burlington, MA, USA) 1 hour before sacrifice.

Alcian blue staining: $5 \mu \mathrm{m}$ thick sections of embedded tumors were cut and adhered to poly-LLysine-coated microscope slides. After dewaxing and rehydration, slides were stained by Alcian blue (pH 1) to visualize PGs, counterstained by nuclear red after which coverslips were fixed. Staining was detected and images were recorded using a slide scanner (X20, Zeiss AxioScan Z1, ZEN software).

Pimonidazole staining: $5 \mu \mathrm{m}$ thick sections of embedded tumors were cut and adhered to poly-LLysine-coated microscope slides. After dewaxing and rehydration of tumor slices, antigens were retrieved (boiling tris: $10 \mathrm{mM}, \mathrm{pH}$ 6) and slides were placed into the InsituPro VSi robot (Intavis bioanalytical instruments, Köln, Germany). Endogenous peroxidases were quenched $\left(\mathrm{H}_{2} \mathrm{O}_{2}: 0.3 \%, 30\right.$ min) and saturated (BSA: $1 \%, 1 \mathrm{~h})$. Slides were then incubated with a primary antibody (antipimonidazole: mouse monoclonal antibody, 1/300, 1h, RT; Hypoxyprobe $\left.{ }^{\mathrm{TM}}\right)$. Biotinylated secondary antibody (1/500, BSA $0.1 \%, 1 \mathrm{~h}$; Vector Laboratories) was used to reveal the first antibody. Biotin was then complexed with streptavidin-coupled horseradish peroxidase (HRP) (1/500, $30 \mathrm{~min}$; Vector Laboratories). Peroxidase was detected using tyramide signal amplification (TSA) as per manufacturer's instructions (TSA-Alexa488; Invitrogen ${ }^{\mathrm{TM}}$ ). Then, nuclei were counterstained with Hoechst (33342) and coverslips were fixed. Emitted fluorescences (461 nm for Hoechst and $519 \mathrm{~nm}$ for TSA) were imaged and recorded as previously (X20, Zeiss AxioScan Z1, ZEN software, Oberkochen, Germany). 
GAG assay: samples $(\mathrm{n}=10)$ were weighed $(20-50 \mathrm{mg})$ and incubated overnight at $65^{\circ} \mathrm{C}$ in $1 \mathrm{~mL}$ of a papain extraction buffer and then centrifuged $(1000 \mathrm{~g}, 10 \mathrm{~min})$. Sulfated GAG content was determined using a Blyscan® Sulfated Glycosaminoglycan assay kit (Biocolor Ltd, Carrickfergus, UK) as per manufacturer's instructions. Absorbance was measured at $656 \mathrm{~nm}$ using Thermo Scientific Multiskan GO device (Thermo Fisher Scientific, Waltham, MA, USA). Experiments were performed in duplicates.

\subsection{Statistical analyses}

Statistical analyses were performed on Prism software version 6.0 (GraphPad Software, San Diego, CA, USA). For in vivo and ex vivo studies, data were presented as individual values with mean \pm SD. Data normal distributions were verified with Shapiro-Wilk tests before performing Student's $t$-tests. For in vivo imaging quantification, paired Student's $t$-tests were applied and for ex vivo analyses, unpaired Student's $t$-tests were performed. Results were considered significant at $P<0.05\left({ }^{*} P<0.05, * * P<\right.$ $0.01, * * * P<0.001, * * * * P<0.0001)$. Significance of results was verified with a Wilcoxon matchedpairs signed rank test.

\section{RESULTS}

\subsection{GAG, guanidyl and APT CEST on phantoms}

GAG CEST effects were observed on the z-spectra of chondroitin 4-sulfate solutions (Figure 1A) and guanidyl and APT CEST effects on that of protamine solutions (Figure 1C). The highest CEST effect offset for each moiety appeared at different chemical shifts: $0.75,1.96$ and 3.68 ppm for GAGs, guanidyl and APT, respectively.

Linear relationships were found between CEST AUCs and either the GAG concentration $\left(\mathrm{R}^{2}=0.9845\right)$ or the $\mathrm{pH}\left(\mathrm{R}^{2}=0.8849\right.$ and $\mathrm{R}^{2}=0.8477$, for Guanidyl* and $\mathrm{APT}^{*}$ respectively $)$. An increase in GAG concentration and in $\mathrm{pH}$ led to an increase of the CEST signal (Figure 1B, 1D). The relative variation of the ratio (reference $\mathrm{pH}$ at 7.2) increased from $\mathrm{pH} 6.8$ to 7.7.

\section{***Figure 1 appears near here $* * *$}

\subsection{Quantitative assessment of GAGs in CHS tumoral tissue}

From signal analysis of GAGs by CEST MRI, a GAG concentration-related map (Figure 2A) was constructed. The signal from GAG was clearly evidenced within tumoral tissue, and was observed to be heterogeneously distributed. Regarding muscle, CEST MRI of GAGs was very low with AUC $=2.15 \pm$ 1.88 as compared to $27.29 \pm 8.38$ in tumor $(P<0.0001 ; n=9)$ (Figure $2 \mathrm{~B}$ ) leading to a tumor/muscle ratio of $23.35 \pm 16.97$

Nuclear imaging, evidenced a significantly $(P<0.0001 ; n=9)$ higher uptake of the ${ }^{99 m}$ Tc-NTP $15-5$ radiotracer in tumoral tissue $(179.38 \pm 38.39 \mathrm{kBq} / \mathrm{mL})$ as opposed to muscle $(35.07 \pm 5.17 \mathrm{kBq} / \mathrm{mL})$, leading to a tumor/muscle ratio of $5.11 \pm 0.69$ (Figure $2 \mathrm{C}$ and 2D). Four images of ${ }^{99 \mathrm{~m} T c-N T P} 15-5$ SPECT imaging are shown on supporting Figure S3A. 
Ex vivo experiments confirmed this high PG feature of CHS tissue. From biochemical assays, GAG concentration was demonstrated to be $6.33 \pm 1.60 \mu \mathrm{g} / \mathrm{mg}$ in the tumor while it was only $0.41 \pm 0.09$ $\mu \mathrm{g} / \mathrm{mg}$ in the muscle $(P<0.0001)$ (Figure $2 \mathrm{~F})$. As illustrated on figure $2 \mathrm{E}$, tumoral tissue was strongly stained by alcian blue confirming its richness in GAGs.

\section{$* * *$ Figure 2 appears near here $* * *$}

\subsection{Quantitative assessment of $\mathrm{pH}$ and hypoxia in CHS tumoral tissue}

Figures $3 \mathrm{~A}$ shows both the anatomical and relative variation of the $\mathrm{APT}^{*} /$ guanidyl* ratio images for 3 randomly chosen mice out of 7 . The ratio was only calculated inside the tumor as it cannot be compared with muscle due to extra CEST signal coming from creatine and phosphocreatine. The CESTbased images highlighted a heterogeneous $\mathrm{pH}$ distribution inside the tumor.

Figure 3B displays a representative image of ${ }^{18} \mathrm{~F}$-FMISO PET. While on this slice, a clear difference between the tumor and the muscle was observed, this was no longer the case once the signal was integrated over the whole tumor (red dots on Figure 3C). At the group level ( $\mathrm{n}=5)$, the tumor mean signal $(18.70 \pm 10.54 \mathrm{kBq} / \mathrm{mL})$ was different from the muscle's signal $(8.60 \pm 4.30 \mathrm{kBq} / \mathrm{mL})$. However, the inter-individual variations, especially for the tumor, led to the absence of differences between both tissues. The three incidences of ${ }^{18} \mathrm{~F}$-FMISO PET imaging are shown on supporting Figure S3B.

Ex vivo experiments by immunofluorescence with pimonidazole evidenced a weak staining within tumoral tissue, mainly localized in the center of CHS lobules (Figure 3D).

\section{$* * *$ Figure 3 appears near here $* * *$}

\section{DISCUSSION}

This work aimed to develop in one-scan multiple CEST MRI contrasts to characterize in vivo PG and hypoxic features of the CHS microenvironment. By exploiting the CEST frequency selectivity, we aimed to simultaneously image the PG concentration and $\mathrm{pH}$ as it relates to hypoxia. CEST MRI images were compared to current state of the art imaging approaches, i.e. ${ }^{99 \mathrm{~m} T c-N T P} 15-5$ and ${ }^{18} \mathrm{~F}-\mathrm{FMISO}$ to map PGs and hypoxia, respectively. To our knowledge, this work is the first one to investigate the microenvironment of CHS by CEST MRI. In most published studies exploiting CEST MRI to image GAG concentration and $\mathrm{pH}$, different saturation protocols are used. Both level and duration of the saturation RF trains are optimized depending on the exchangeable protons targeted. Typically, to image the hydroxyl moieties, the saturation pulse level is moderate while the duration is long. ${ }^{(39,40)}$ For APT CEST, a variety of saturation conditions have been used. ${ }^{(41)}$ However, to maximize the APT effect, Zhou et al. ${ }^{(41)}$ recommend the usage of a saturation duration higher than $0.8 \mathrm{~s}$ and a level of around $2 \mu \mathrm{T}$. Our experimental conditions were close to the optimal ones for the different contrasts. As we wanted to image both tumor features in a single acquisition, we chose to use a long saturation train $\left(t_{\text {sat }}=4 \mathrm{~s}\right)$ with moderate power $\left(\mathrm{B}_{1}=1.5 \mu \mathrm{T}\right)$ that was demonstrated from phantom experiments to be a satisfactory compromise. Indeed, the GAG CEST effect was not too broad and did not overlap with the guanidyl 
301

302

303

304

305

306

307

308

309

310

311

312

313

314

315

316

317

318

319

320

321

322

323

324

325

326

327

328

329

330

331

332

333

334

335

336

337

one. Obviously, this compromise on the saturation parameters is at the cost of the optimized sensitivity for each CEST effect. Carefully choosing the saturation frequencies made it possible to simultaneously assess PGs (GAG CEST) and pH (guanidyl and APT CEST). In vitro, we can note that we found an increase of the guanidyl* signal like Oskolkov et al. ${ }^{(42)}$ while Jin et al. ${ }^{(34)}$ observed an opposite trend. This discrepancy can be due to (i) our multiple-contrast CEST conditions, experimental conditions were not set to the optimal values to maximize the CEST effect for $\mathrm{pH}$, (ii) physical parameters, especially the chemical exchange rate constant, are not comparable to what was simulated in the other study. To obtain a $\mathrm{pH}$ variation independent of protein concentration, the ratio of the APT* and guanidyl* CEST was calculated.

In vivo GAG CEST images were obtained by acquiring signals resulting from the chemical exchange of the abundant hydroxyl groups in GAGs. A 23 times higher CEST effect was observed for the tumor compared to muscle demonstrating the high PG density in the CHS microenvironment. CEST signal studies of different forms of GAGs typically found in articular cartilage recently demonstrated that the CEST effect arises mainly from chondroitin 4-sulfate. ${ }^{(43)}$ SRC was demonstrated to highly express aggrecan which is composed of a core protein with covalently attached side chains of linear GAGs, including a high number of chondroitin sulfate, especially chondroitin 4-sulfate. ${ }^{(44)}$ The GAG CEST approach was combined with ${ }^{99 \mathrm{mTc}-\mathrm{NTP}}$ 15-5 nuclear imaging, a radiotracer designed to target negatively charged molecules of GAGs. ${ }^{(16,45)}$ As expected, a higher radiotracer accumulation was observed in tumor in respect to muscle (ratio $\sim 5$ ), confirming the higher PG concentration measured by GAG CEST. The discrepancies in the tumor to muscle ratios between the MRI and nuclear imaging can be explained by the poor vascularization of the tumor, thereby limiting the delivery of the radiotracer. Since CEST MRI provided signals from hydroxyl moieties of PGs, we also have to consider that hydroxyl moieties are found in other molecules such as glucose or lactate. ${ }^{(46,47)}$ We therefore were interested to complete GAG CEST with ${ }^{1} \mathrm{H}$ MRS to identify their respective contribution in the CEST signal. Unfortunately, due to its high chondrogenic structure, CHS tissue did not provide enough resolution on the MRS spectrum. Finally, ex vivo quantitative analysis, i.e. GAG assays, reinforced the in vivo multimodal imaging results and evidenced high PG concentration within tumoral tissue (ratio tumor/muscle $\sim 18$, i.e. in between SPECT and CEST ratios).

CEST MRI assessment of intratumoral $\mathrm{pH}$ variations was performed by exploiting both guanidyl and amide to enhance the sensitivity of $\mathrm{pH}$ weighted MRI. ${ }^{(34)}$ As acidosis is associated to hypoxia ${ }^{(48)}$, it was expected that mapping tumoral $\mathrm{pH}$ through CEST MRI would be a relevant methodology to delineate areas of hypoxic and well oxygenated cells within tumoral tissue. The CEST signals from both chemical groups were assigned to proteins. To have a map independent from protein concentration, the APT*/guanidyl* ratio was calculated inside the tumor. This approach assumes that the guanidyl signal is coming only from the proteins. This hypothesis is acceptable for tumors, although it cannot be applied to other tissues, especially muscle, due to the presence of creatine and phosphocreatine. These metabolites give a guanidyl signal, the APT*/guanidyl* ratio will depend on both $\mathrm{pH}$ and the metabolite 
338

339

340

341

342

343

344

345

346

347

348

349

350

351

352

353

354

355

356

357

358

359

360

361

362

363

364

365

366

367

368

369

370

371

372

373

concentrations. In vivo ${ }^{18} \mathrm{~F}$-FMISO PET imaging confirmed the hypoxic status of CHS since the mean tumor/muscle ratio value was approximately 2 . However, at the group level there was no statistical difference between the two tissues due to large inter-individual variations. Ex vivo immunofluorescence with pimonidazole evidenced that hypoxic areas were heterogeneously distributed throughout the tumoral tissue similarly to CEST MRI.

The assessment of pH by APT CEST is debated in the literature. Some authors reported a decrease of the CEST effect on the $\mathrm{pH}^{(49,50)}$ while others observed the opposite. ${ }^{(51,52)}$ Most of these APT CEST effects were obtained after performing the so-called $\mathrm{MTR}_{\text {asym }}$ analysis. The key assumption in this data processing is that for negative offsets, only the direct water saturation effect is recorded. ${ }^{(53)}$ However, it was shown in vivo that several NOE exchanges were recorded in the region of $\sim-1$ to $-4 \mathrm{ppm}$, no longer fulfilling the $\mathrm{MTR}_{\text {asym }}$ assumption. ${ }^{(54)}$ Nevertheless, most of these studies used this estimator. To avoid the interference of the NOE on our CEST quantifications, our data were analyzed by using the approach inspired by Jin et al. ${ }^{(20)}$ and described in section 2.3.1.

To conclude, our work on SRC bearing mice demonstrated the potential of imaging the different tumoral microenvironment properties by a single CEST MRI acquisition. MRI presents the main advantage when compared to nuclear imaging to give high-resolution spatial information. It is then possible to delineate accurately the metabolism-related region of interest. Such parametric maps would allow a better understanding of the relationship between tumor cells and their environment, thus becoming a prerequisite to develop more individualized therapies.

ACKNOWLEDGEMENTS The authors thank Christelle Damon-Soubeyrand (Anipath facility) for her precious technical help with histological equipment.

\section{REFERENCES}

1. Key Statistics for Bone Cancer: Key Statistics About Bone Cancer. American Cancer Society Web site. https://www.cancer.org/cancer/bone-cancer/about/key-statistics.html. Updated January 12, 2021. Accessed January 22, 2021.

2. World Health Organization WHO, Fletcher C, Bridge JA, Hogendoorn PCW, Mertens F. WHO Classification of Tumours of Soft Tissue and Bone. In: WHO Classification of Tumours, vol. 5. 4th ed. World Health Organization; 2013. p 264-268.

3. Gelderblom H, Hogendoorn PCW, Dijkstra SD, et al. The clinical approach towards chondrosarcoma. Oncologist. 2008;13(3):320-329.

4. Mery B, Espenel S, Guy J-B, et al. Biological aspects of chondrosarcoma: Leaps and hurdles. Crit Rev Oncol Hemat. 2018;126.

5. Angelini A, Guerra G, Mavrogenis AF, Pala E, Picci P, Ruggieri P. Clinical outcome of central conventional chondrosarcoma. J Surg Oncol. 2012;106(8):929-937. 
6. van Oosterwijk JG, Anninga JK, Gelderblom H, Cleton-Jansen A-M, Bovée JVMG. Update on targets and novel treatment options for high-grade osteosarcoma and chondrosarcoma. Hematol Oncol Clin North Am. 2013;27(5):1021-1048.

7. Nazeri E, Gouran Savadkoohi M, Majidzadeh-A K, Esmaeili R. Chondrosarcoma: An overview of clinical behavior, molecular mechanisms mediated drug resistance and potential therapeutic targets. Crit Rev Oncol Hematol. 2018;131:102-109.

8. Yeldag G, Rice A, Del Río Hernández A. Chemoresistance and the self-maintaining tumor microenvironment. Cancers (Basel). 2018;10(12):471.

9. Davies C de L, Berk DA, Pluen A, Jain RK. Comparison of IgG diffusion and extracellular matrix composition in rhabdomyosarcomas grown in mice versus in vitro as spheroids reveals the role of host stromal cells. Br J Cancer. 2002;86(10):1639-1644.

10. Trédan O, Galmarini CM, Patel K, Tannock IF. Drug resistance and the solid tumor microenvironment. J Natl Cancer Inst. 2007;99(19):1441-1454.

11. Skeletal Lesions Interobserver Correlation among Expert Diagnosticians (SLICED) Study Group. Reliability of histopathologic and radiologic grading of cartilaginous neoplasms in long bones. J Bone Joint Surg Am. 2007;89(10):2113-2123.

12. Murphey MD, Walker EA, Wilson AJ, Kransdorf MJ, Temple HT, Gannon FH. From the archives of the AFIP: imaging of primary chondrosarcoma: radiologic-pathologic correlation. Radiographics. 2003;23(5):1245-1278.

13. Logie CI, Walker EA, Forsberg JA, Potter BK, Murphey MD. Chondrosarcoma: A diagnostic imager's guide to decision making and patient management. Semin Musculoskelet Radiol. 2013;17(2):101-115.

14. Jo I, Gould D, Schlicht S, Taubman K, Choong P. Diagnostic accuracy of functional imaging modalities for chondrosarcoma: A systematic review and meta-analysis. $J$ Bone Oncol. 2019;19:100262.

15. Miot-Noirault E, Gouin F, Vidal A, et al. First preclinical imaging of primary cartilage neoplasm and its local recurrence using 99mTc-NTP 15-5 radiotracer. J Nucl Med. 2009;50(9):1541-1547.

16. Peyrode C, Gouin F, Vidal A, et al. A"Proteoglycan targeting strategy" for the scintigraphic imaging and monitoring of the swarm rat chondrosarcoma orthotopic model. Sarcoma. 2011;2011:691608.

17. Miot-Noirault E, Vidal A, Auzeloux P, Peyrode C, Madelmont J-C, Chezal J-M. In vivo scintigraphic imaging of proteoglycans. Methods Mol Biol. 2012;836:183-198.

18. Rajendran JG, Mankoff DA, O'Sullivan F, et al. Hypoxia and glucose metabolism in malignant tumors: Evaluation by [18F]Fluoromisonidazole and [18F]Fluorodeoxyglucose positron emission tomography imaging. Clin Cancer Res. 2004;10(7):2245-2252.

19. Goldenberg JM, Pagel MD. Assessments of tumor metabolism with CEST MRI. NMR Biomed. 2019;32(10):e3943. 
411

412

413

414

415

416

417

418

419

420

421

422

423

424

425

426

427

428

429

430

431

432

433

434

435

436

437

438

439

440

441

442

443

444

445

446

20. Jin T, Wang P, Zong X, Kim S-G. MR imaging of the Amide-Proton Transfer effect and the $\mathrm{pH}-$ insensitive Nuclear Overhauser Effect at 9.4 T. Magn Reson Med. 2013;69(3):760-770.

21. Zhou Y, van Zijl PCM, Xu X, et al. Magnetic resonance imaging of glycogen using its magnetic coupling with water. Proc Natl Acad Sci USA. 2020;117(6):3144-3149.

22. Jones KM, Pollard AC, Pagel MD. Clinical applications of chemical exchange saturation transfer (CEST) MRI. J Magn Reson Imaging. 2018;47(1):11-27.

23. Kogan F, Hariharan H, Reddy R. Chemical Exchange Saturation Transfer (CEST) imaging: Description of technique and potential clinical applications. Curr Radiol Rep. 2013;1(2):102114.

24. van Zijl PCM, Yadav NN. Chemical Exchange Saturation Transfer (CEST): what is in a name and what isn't? Magn Reson Med. 2011;65(4):927-948.

25. Wu B, Warnock G, Zaiss M, et al. An overview of CEST MRI for non-MR physicists. EJNMMI Phys. 2016;3(1):19.

26. Dou W, Lin C-YE, Ding H, et al. Chemical exchange saturation transfer magnetic resonance imaging and its main and potential applications in pre-clinical and clinical studies. Quant Imaging Med Surg. 2019;9(10):1747-1766.

27. Ling W, Regatte RR, Navon G, Jerschow A. Assessment of glycosaminoglycan concentration in vivo by chemical exchange-dependent saturation transfer (gagCEST). Proc Natl Acad Sci USA. 2008;105(7):2266-2270.

28. Brinkhof S, Nizak R, Khlebnikov V, Prompers JJ, Klomp DWJ, Saris DBF. Detection of early cartilage damage: feasibility and potential of gagCEST imaging at 7T. Eur Radiol. 2018;28(7):2874-2881.

29. Krusche-Mandl I, Schmitt B, Zak L, et al. Long-term results 8 years after autologous osteochondral transplantation: $7 \mathrm{~T}$ gagCEST and sodium magnetic resonance imaging with morphological and clinical correlation. Osteoarthr Cartil. 2012;20(5):357-363.

30. Krishnamoorthy G, Nanga RPR, Bagga P, Hariharan H, Reddy R. High Quality 3D gagCEST Imaging of In Vivo Human Knee Cartilage at 7T. Magn Reson Med. 2017;77(5):1866-1873.

31. Wei W, Lambach B, Jia G, Kaeding C, Flanigan D, Knopp MV. A Phase I clinical trial of the knee to assess the correlation of gagCEST MRI, delayed gadolinium-enhanced MRI of cartilage and T2 mapping. Eur J Radiol. 2017;90:220-224.

32. Corbet C, Feron O. Tumour acidosis: from the passenger to the driver's seat. Nat Rev Cancer. 2017;17(10):577-593.

33. Chen LQ, Pagel MD. Evaluating $\mathrm{pH}$ in the extracellular tumor microenvironment using CEST MRI and other imaging methods. Adv Radiol. 2015;2015:1-25.

34. Jin T, Wang P, Hitchens TK, Kim S-G. Enhancing sensitivity of pH-weighted MRI with combination of amide and guanidyl CEST. Neuroimage. 2017;157:341-350. 
35. Maloney E, Wang Y-N, Vohra R, et al. Magnetic resonance imaging biomarkers for pulsed focused ultrasound treatment of pancreatic ductal adenocarcinoma. World J Gastroenterol. 2020;26(9):904-917.

36. Loi L, Zimmermann F, Goerke S, et al. Relaxation-compensated CEST (chemical exchange saturation transfer) imaging in breast cancer diagnostics at 7T. Eur J Radiol. 2020;129:109068.

37. Kim M, Gillen J, Landman BA, Zhou J, van Zijl PCM. Water saturation shift referencing (WASSR) for chemical exchange saturation transfer (CEST) experiments. Magn Reson Med. 2009;61(6):1441-1450.

38. Vidal A, Gaumet V, Galmier M-J, et al. Development of a freeze-dried kit formulation for the preparation of 99m Tc-NTP 15-5, a radiotracer for scintigraphic imaging of proteoglycans. Appl Radiat Isot. 2015;101:1-9.

39. Zhou Z, Bez M, Tawackoli W, et al. Quantitative chemical exchange saturation transfer MRI of intervertebral disc in a porcine model. Magn Reson Med. 2016;76(6):1677-1683.

40. Nasrallah FA, Pagès G, Kuchel PW, Golay X, Chuang K-H. Imaging brain deoxyglucose uptake and metabolism by glucoCEST MRI. J Cereb Blood Flow Metab. 2013;33(8):1270-1278.

41. Zhou J, Heo H-Y, Knutsson L, van Zij1 PCM, Jiang S. APT-Weighted MRI: techniques, current neuro applications, and challenging issues. J Magn Reson Imaging. 2019;50(2):347-364.

42. Oskolkov N, Bar-Shir A, Chan KWY, et al. Biophysical characterization of human protamine-1 as a responsive CEST MR Contrast Agent. ACS Macro Lett. 2015;4(1):34-38.

43. Einarsson E, Peterson P, Önnerfjord P, et al. The role of cartilage glycosaminoglycan structure in gagCEST. NMR Biomed. 2020;33(5):e4259.

44. Choi HU, Meyer K, Swarm R. Mucopolysaccharide and protein-polysaccharide of a transplantable rat chondrosarcoma. Proc Natl Acad Sci USA. 1971;68(5):877-879.

45. Miot-Noirault E, Gouin F, Vidal A, et al. First preclinical imaging of primary cartilage neoplasm and its local recurrence using 99mTc-NTP 15-5 radiotracer. J Nucl Med. 2009;50(9):1541-1547.

46. Walker-Samuel S, Ramasawmy R, Torrealdea F, et al. In vivo imaging of glucose uptake and metabolism in tumors. Nat Med. 2013;19(8):1067-1072.

47. DeBrosse C, Nanga RPR, Bagga P, et al. Lactate Chemical Exchange Saturation Transfer (LATEST) Imaging in vivo A Biomarker for LDH Activity. Sci Rep. 2016;6:19517.

48. Chiche J, Brahimi-Horn MC, Pouysségur J. Tumour hypoxia induces a metabolic shift causing acidosis: a common feature in cancer. J Cell Mol Med. 2010;14(4):771-794.

49. Schüre J, Shrestha M, Breuer S, et al. The pH sensitivity of APT-CEST using phosphorus spectroscopy as a reference method. NMR Biomed. 2019;32(11).

50. Wang E, Wu Y, Cheung JS, et al. Mapping tissue $\mathrm{pH}$ in an experimental model of acute stroke Determination of graded regional tissue $\mathrm{pH}$ changes with non-invasive quantitative amide proton transfer MRI. Neuroimage. 2019;191:610-617. 
483 51. Krikken E, van der Kemp WJM, Khlebnikov V, et al. Contradiction between amide-CEST signal 484 and $\mathrm{pH}$ in breast cancer explained with metabolic MRI. NMR Biomed. 2019;32(8).

52. Schmidt H, Schwenzer NF, Gatidis S, et al. Systematic evaluation of amide proton Chemical Exchange Saturation Transfer at $3 \mathrm{~T}$ : Effects of protein concentration, $\mathrm{pH}$, and acquisition parameters. Invest Radiol. 2016;51(10):635-646.

488 53. Liu G, Song X, Chan KWY, McMahon MT. Nuts and bolts of chemical exchange saturation 489 transfer MRI. NMR Biomed. 2013;26(7):810-828.

54. Zhang X-Y, Wang F, Li H, et al. Accuracy in the quantification of chemical exchange saturation 492 transfer (CEST) and relayed nuclear Overhauser enhancement (rNOE) saturation transfer effects. NMR Biomed. 2017;30(7):e3716. 
494

495

496

497

498

499

500

501

502

503

504

505

506

507

508

509

510

511

512

513

514

515

516

517

518

519

520

\section{FIGURE CAPTIONS}

FIGURE 1: z-spectra of (A) chondroitin 4-sulfate and (C) protamine at several concentrations and $\mathrm{pH}$, respectively. The CEST effects were estimated by measuring the AUC from 0.02 to $1.50 \mathrm{ppm}$ for chondroitin 4-sulfate (GAG CEST) and the signal intensity at 1.96 and 3.60 for guanidyl* and APT* CEST, respectively. The relationship between CEST quantification and concentrations or $\mathrm{pH}$ are represented on panels (B) and (D), respectively.

FIGURE 2: In vivo multimodal imaging of GAGs in SRC bearing mice (A) GAG CEST parametric image. To clearly identify the tumor, the high-resolution image was added inside the inset. (C) SPECTCT imaging with ${ }^{99 m}$ Tc-NTP 15-5. ROI quantitative analysis of GAG CEST and SPECT-CT are presented in (B) and (D), respectively. (E) Ex vivo tumor slice (X20 magnification) stained with both alcian blue and nuclear red. Data were presented by individual values with mean $\pm \mathrm{SD}$. Paired t-test and unpaired t-test $(* * * * P<0.0001)$ were applied for in vivo and ex vivo quantifications, respectively. Red data points on panels (B), (D) and (F) correspond to the images shown in (A), (C) and (E), respectively. Abbreviations: T: Tumor; M: muscle.

FIGURE 3: In vivo multimodal imaging of $\mathrm{pH}$ acidification (MRI) and hypoxia (PET) in SRC bearing mice. (A) High resolution and relative variation of APT*/guanidyl* ratio images for 3 representative mice. (B) PET imaging with ${ }^{18} \mathrm{~F}$-FMISO. ROI quantitative analysis of PET is presented in (C). (D) Ex vivo tumor slice (X20 magnification) stained with pimonidazole and Hoechst. Data were presented by individual values with mean $\pm \mathrm{SD}$. Paired t-test $(* P=0.0164)$ were applied for in vivo quantifications. Red data points on panels $(\mathrm{C})$ correspond to the image shown in (B). Abbreviations: T: Tumor; M: muscle. 


\section{SUPPORTING FIGURE AND TABLE CAPTIONS}

522

523

Supporting Table S1: Overview of the experimental design and analysis by in vivo modality.

524

525 Supporting Figure S1: Tumoral volumes measured by MRI at 15-16 days post-induction and presented

526 as individual values with mean $\pm \mathrm{SD}$. Averaged volume $215.04 \pm 86.28 \mathrm{~mm}^{3}$.

527

528 Supporting Figure S2: Representative data obtained in vivo for a ROI positioned in (blue) the tumor 529 and (red) the muscle. (A) Mean z-spectra for all animals; dotted lines represent \pm 1 SD. (B) z-spectra of 530 a representative animal; dotted lines represent the linear baselines applied for guanidyl (from 1.66 to $5312.30 \mathrm{ppm}$ ) and APT CEST (from 3.36 to $3.78 \mathrm{ppm}$ ) analyses. (C) Lorentzian fit of the GAG CEST from 532 the z-spectra shown in (B).

533

534 Supporting Figure S3: (A) ${ }^{99 m}$ Tc-NTP 15-5 SPECT-CT image of a representative animal. From left to 535 right: axial slice with tumor, coronal slice with tumor, coronal slice with radiotracer body dispersion 536 and sagittal slice with tumor and radiotracer body dispersion. The areas are annotated T for tumor, M 537 for muscle, B for bladder and Li for liver. (B) ${ }^{18} \mathrm{~F}$-FMISO PET image of a representative animal. From 538 left to right: axial slice with tumor, coronal and sagittal slices with tumor and radiotracer body 539 dispersion. The areas were annotated $\mathrm{T}$ for tumor, $\mathrm{M}$ for muscle, DT for digestive tract. Orientations 540 were annotated A for anterior, D for dorsal, L for left, $\mathrm{P}$ for posterior, $\mathrm{R}$ for right and $\mathrm{V}$ for ventral. 


\section{ABBREVIATIONS}

542

543 APT: Amide proton transfer

544 AUC: Area under the curve

545 BSA: Bovine serum albumin

546 CHS: Chondrosarcoma

547 DMEM: Dulbecco's modified eagle medium

548 DWI: Diffusion-weighted imaging

549 ECM: Extracellular matrix

550 GAG: Glycosaminoglycan

551 HRP: Horseradish peroxidase

552 IVIA: In vivo imaging in Auvergne

553 MTC: Magnetization transfer contrast

554 MTR $_{\text {asym }}$ : Magnetization transfer asymmetry

555 NOE: Nuclear overhauser enhancement

556 OSEM: Ordered subset expectation maximization

557 PBS: Phosphate-buffered saline

558 PG: Proteoglycan

559 RARE: Rapid acquisition with relaxation enhancement

560 ROI: Region of interest

561 SRC: Swarm rat chondrosarcoma

562 TSA: Tyramide signal amplification

563 WASSR: Water saturation shift reference

564

565 
(A)

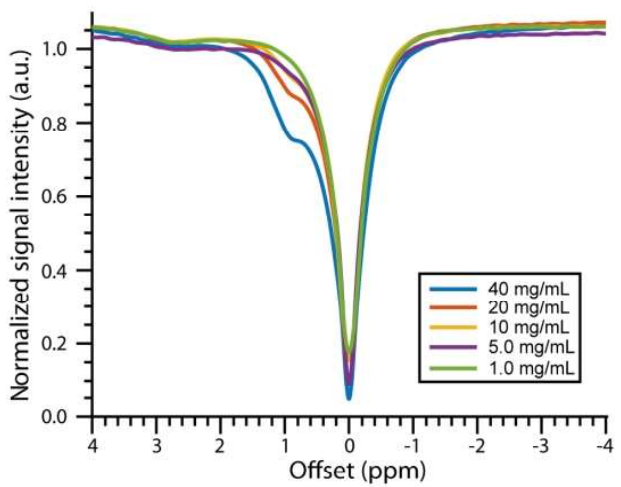

(C)

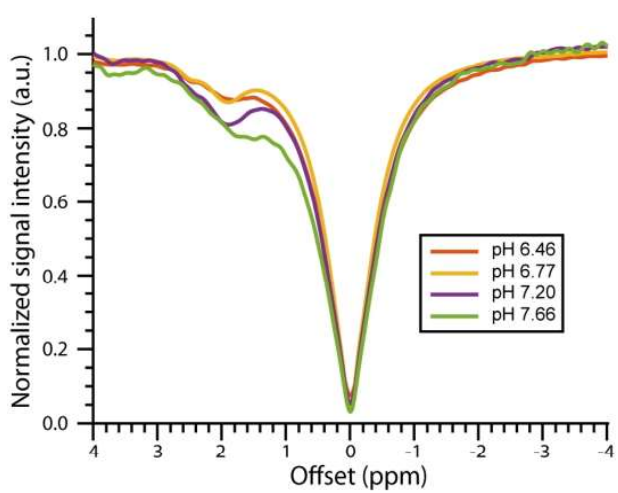

(B)

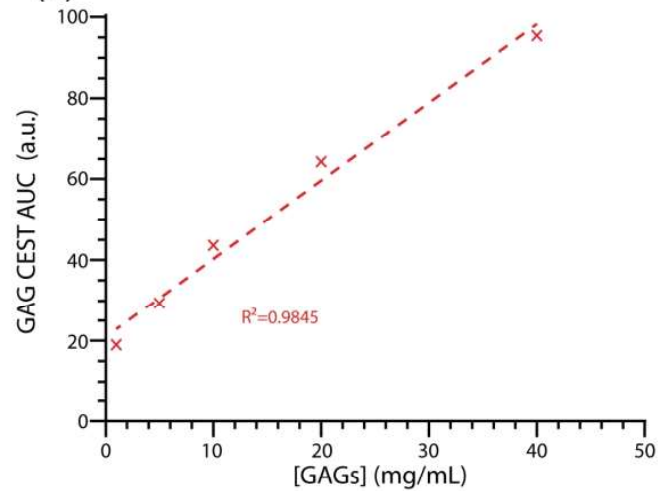

(D)
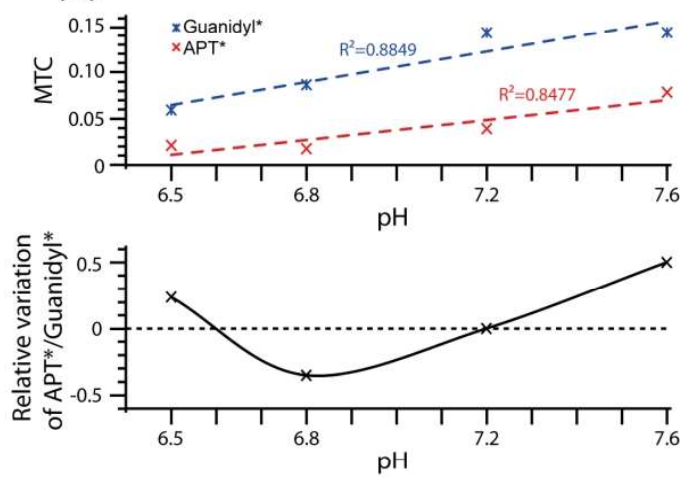

FIGURE 1: $z$-spectra of (A) chondroitin 4-sulfate and (C) protamine at several concentrations and $\mathrm{pH}$, respectively. The CEST effects were estimated by measuring the AUC from 0.02 to 1.50 ppm for chondroitin 4-sulfate (GAG CEST) and the signal intensity at 1.96 and 3.60 for guanidyl* and APT* CEST, respectively. The relationship between CEST quantification and concentrations or $\mathrm{pH}$ are represented on panels (B) and (D), respectively. 
(A)

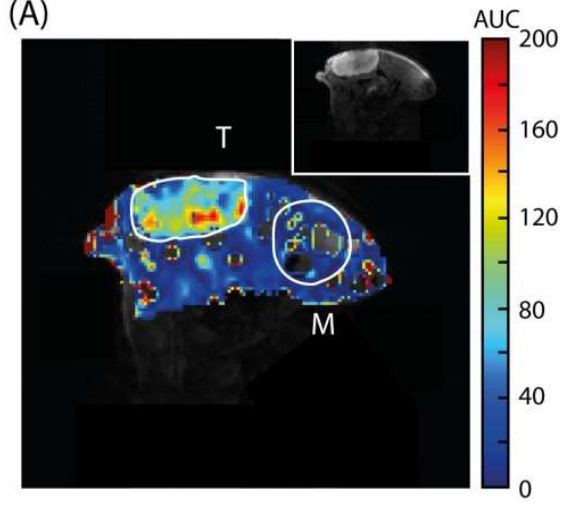

(C)

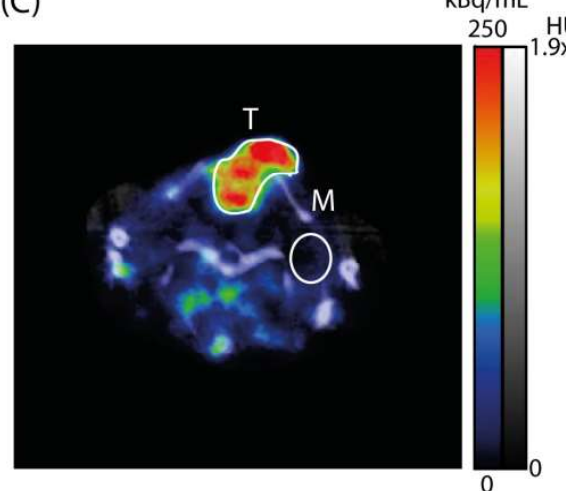

(E)

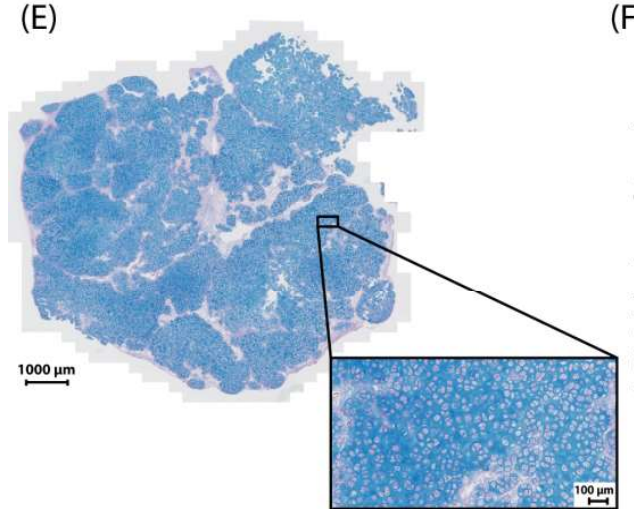

(F)
(B)

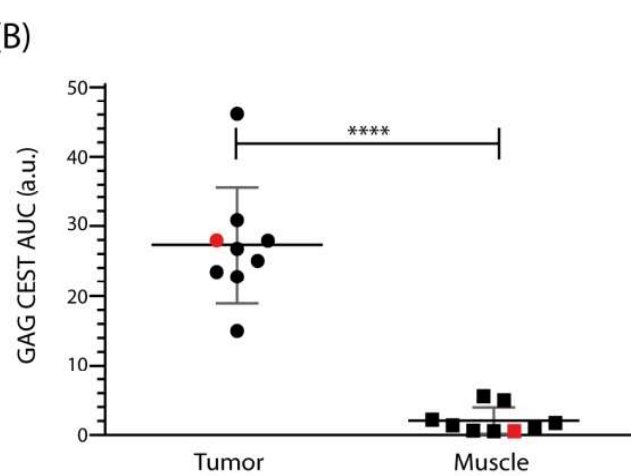

(D)
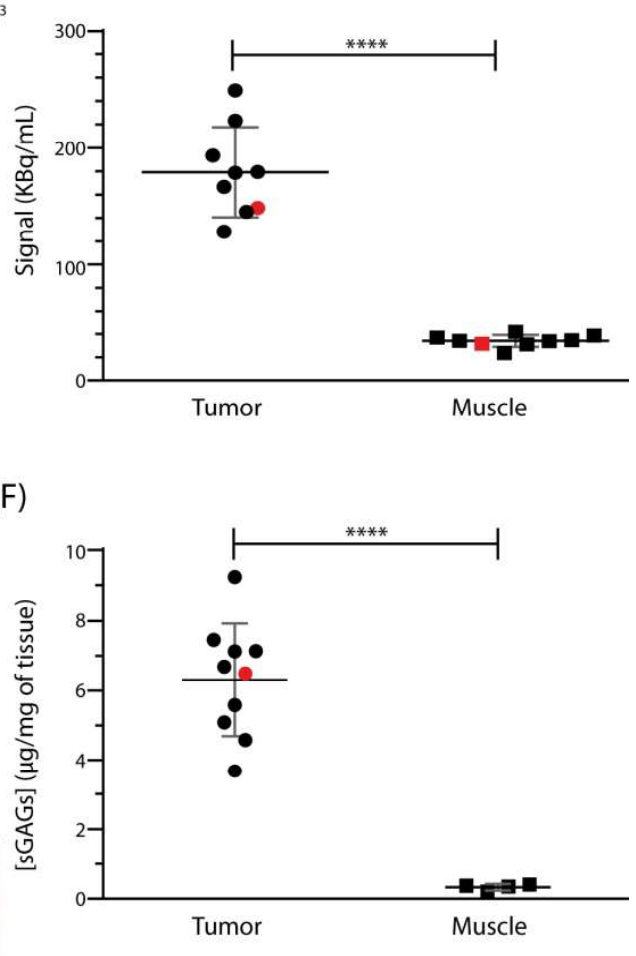

FIGURE 2: In vivo multimodal imaging of GAGs in SRC bearing mice (A) GAG CEST parametric image. To clearly identify the tumor, the high-resolution image was added inside the inset. (C) SPECT-CT imaging with 99mTc-NTP 15-5. ROI quantitative analysis of GAG CEST and SPECT-CT are presented in (B) and (D), respectively. (E) Ex vivo tumor slice (X20 magnification) stained with both alcian blue and nuclear red. Data were presented by individual values with mean \pm SD. Paired t-test and unpaired t-test $(* * * * P<0.0001)$ were applied for in vivo and ex vivo quantifications, respectively. Red data points on panels (B), (D) and (F) correspond to the images shown in (A), (C) and (E), respectively. Abbreviations: T: Tumor; M: muscle. 


\section{(A)}

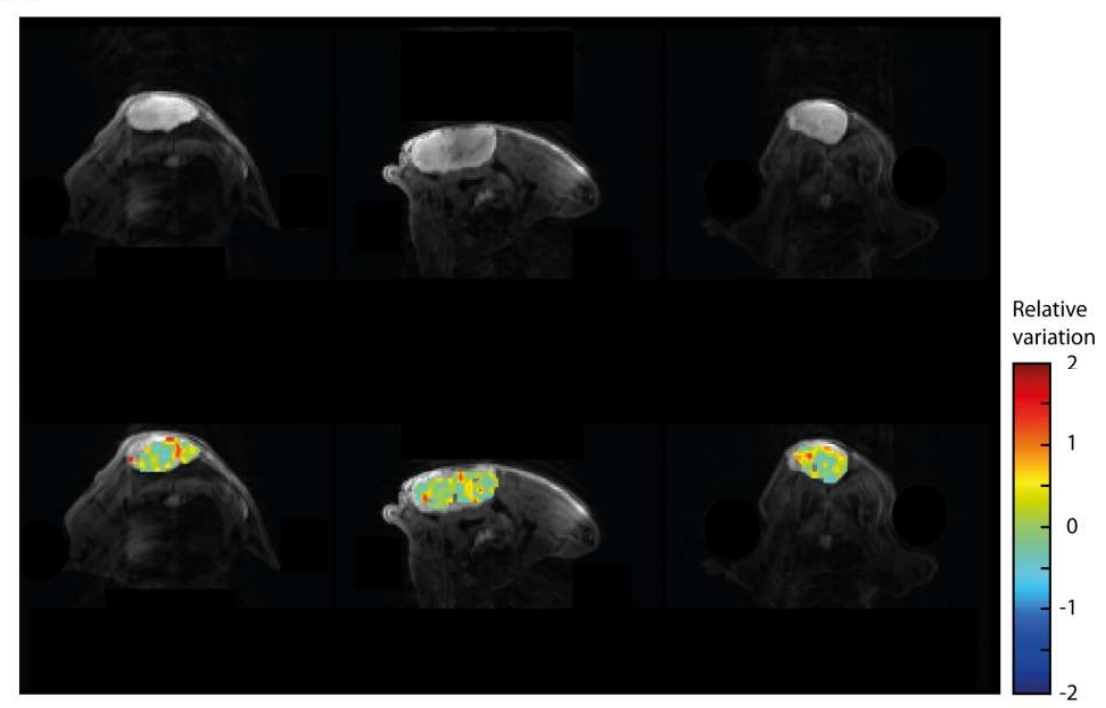

(B)

(C)
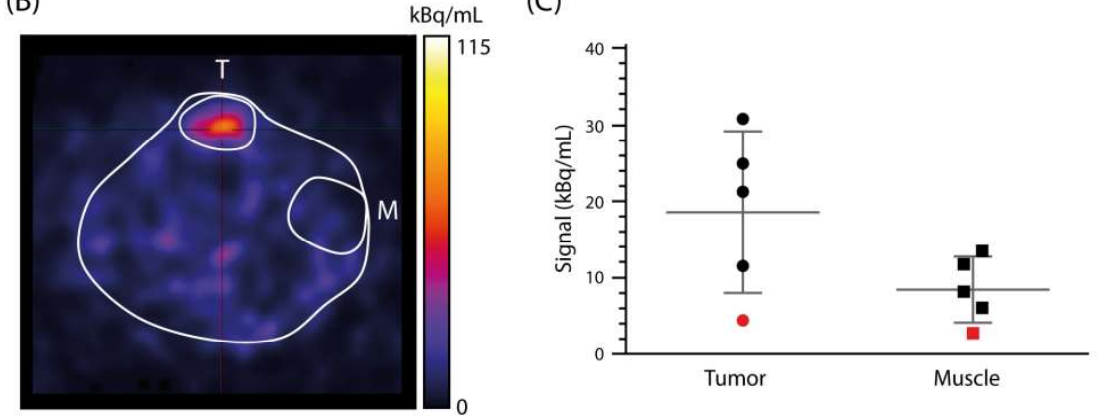

(D)

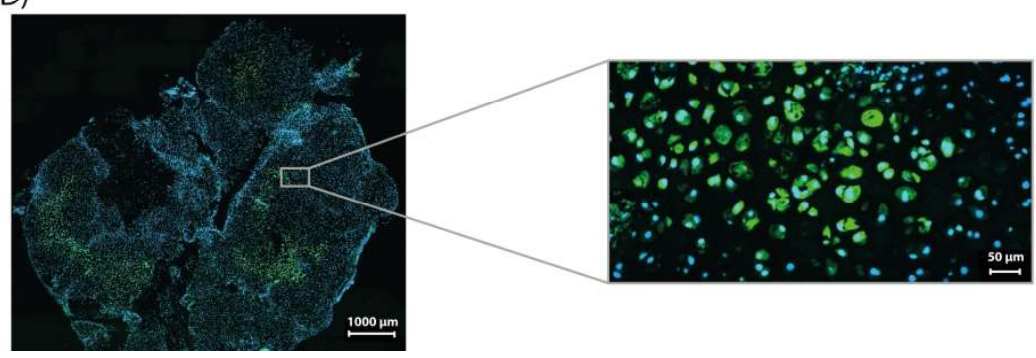

FIGURE 3: In vivo multimodal imaging of $\mathrm{pH}$ acidification (MRI) and hypoxia (PET) in SRC bearing mice. (A) High resolution and relative variation of APT*/guanidyl* ratio images for 3 representative mice. (B) PET imaging with ${ }^{18} \mathrm{~F}$-FMISO. ROI quantitative analysis of PET is presented in (C). (D) Ex vivo tumor slice (X20 magnification) stained with pimonidazole and Hoechst. Data were presented by individual values with mean $\pm \mathrm{SD}$. Paired t-test $(* P=0.0164)$ were applied for in vivo quantifications. Red data points on panels (C) correspond to the image shown in (B). Abbreviations: T: Tumor; M: muscle. 